\title{
Deterministic and Probabilistic Analysis of a Simple Markov Model: How Different Could They Be?
}

\author{
Howard Thom ${ }^{1,2}$ D \\ Accepted: 7 November 2021 / Published online: 20 January 2022 \\ (c) The Author(s) 2022, Corrected Publication 2022
}

\section{Background}

Markov multistate models are among the most common models used for health economic evaluations [1]. These break diseases into a finite set of health states, divide a simulated cohort of patients amongst these states, and simulate their disease by allowing transitions between states over time. They are accepted by healthcare decision makers, including the National Institute for Health and Care Excellence (NICE) and Canadian Agency for Drugs and Technologies in Health (CADTH) [2, 3].

Evidence informing parameters of Markov models, such as state transition probabilities, costs and health-related utilities, is often limited [4]. Probabilistic analysis evaluates the model over a distribution of these parameters and bases decisions on the distribution of outputs; deterministic analysis evaluates the model at parameter means, giving only a single output for decision making. As a Markov model is a nonlinear function, the mean of a probabilistic analysis will not match the output of a deterministic analysis. This follows from the general statement that the expectation of a nonlinear function is not equal to the nonlinear function acting on the expectation, of which Jensen's inequality on convex functions is a special case [5,6]. This was demonstrated in practice in the NICE evaluation of rivaroxaban in coronary or peripheral artery disease. The deterministic incremental cost-effectiveness ratio (ICER) was GBP16,602 per quality adjusted life year (QALY) while the probabilistic ICER was GBP8138/QALY, a factor of two reduction [7]. Wilson made this point and argued that decision makers should use only probabilistic analysis [8]. Furthermore, official guidance

Howard Thom

howard.thom@bristol.ac.uk

1 Bristol Medical School: Population Health Sciences, University of Bristol, Bristol, UK

2 Clifton Insight, Bristol, UK from NICE and CADTH both recommend the use of probabilistic analysis as the base case [2,3].

Despite publicly available examples, theoretical argument, and official guidance, deterministic analyses remain common. Even if the base-case analysis is probabilistic, modellers will often use deterministic sensitivity analyses. The usual response to criticism is "How different could they be?" In this simple simulation study, I will show that they could be very different.

\section{Exploratory Simulation Study}

The simulation study is based on a comparison of two treatments using a discrete-time 3-state cohort Markov model with 6-month cycles and 5-year time horizon. States are either healthy, adverse event, and dead, and all patients begin in the healthy state. The adverse event state has lower health-related utility and higher costs than the healthy state. Treatment 1 is the reference while treatment 2 has lower mortality, more adverse events, and higher cost. In probabilistic analysis, utilities and costs are Normally distributed, probabilities of death and adverse event on treatment 1 are Gamma, and log odds ratios for treatment 2 relative to treatment 1 are Normal. For deterministic analysis, the means of each parameter are used while probabilistic analysis uses 1000 parameter samples.

In the simulation study 10,000 samples from Uniform distributions are used to define the distributions on probabilities and log odds ratios. I refer to each iteration of the simulation as a scenario to avoid confusion with simulations in the probabilistic analysis. Parameters of cost and utilities are held constant.

Under each scenario, cost-effectiveness summaries are calculated and compared between the deterministic and probabilistic analysis. Total costs and total QALYs, discounted at $3.5 \%$ per year, are calculated. Incremental costs, QALYs, and net benefits (INB) at GBP20,000/QALY are 
calculated for treatment 2 relative to treatment 1 , along with the ICER [2]. For probabilistic analysis, the probability that treatment 2 is cost effective at GBP20,000/QALY is calculated, which is a point of the cost-effectiveness acceptability curve (CEAC), as is expected value of perfect information (EVPI) [4].

Full details of the model and simulation are provided in the supplementary material. They were implemented in the $\mathrm{R}$ statistical programming language and code is in the supplementary material $[9,10]$.

\section{Results of Simulation Study}

Comparison of conclusions under the two analyses are presented in detail in supplementary material. In brief, if treatment 1 is dominant under probabilistic analysis, it is also dominant under deterministic analysis in $99 \%$ of scenarios. If treatment 1 is only cost-effective and not dominant under probabilistic analysis, the deterministic analysis finds treatment 2 to be dominant or cost-effective in a small number of scenarios (1.8\%). If treatment 2 is cost-effective but not dominant under probabilistic analysis, treatment 1 is dominant or cost-effective under deterministic analysis in a larger percentage of scenarios (6.9\%).

Across scenarios, probabilistic INBs are similar to deterministic INBs (Fig. 1). The mean and 95\% reference range under deterministic analysis was $£ 6,021.82(-4359.20$, 17707.67), under probabilistic £6,028.64 (- 4363.49, 17688.01), and probabilistic minus deterministic $£ 6.82$ $(-264.20,243.35)$.

Results of the two worst case scenarios are tabulated in supplementary material. In a maximum ICER difference scenario, deterministic analysis found treatment 2 not costeffective with ICER of $£ 23,536 / \mathrm{Q} A L Y$ while probabilistic analysis found treatment 2 cost-effective with an ICER of $£ 19,661 / \mathrm{Q} A L Y$. In a maximum CEAC inconsistency scenario, deterministic and probabilistic analysis found treatment 2 not cost-effective with ICERs of $£ 30,226 /$ QALY and $£ 26,382 / \mathrm{QALY}$, respectively. However, the probabilistic analysis found $45 \%$ of simulations below $£ 20,000$ /QALY, indicating substantial uncertainty.

\section{How Different Could They Be?}

Our simple analysis of a 3-state Markov model found that results, and indeed conclusions, of deterministic and probabilistic analysis can be different. They disagreed on costeffectiveness in $2-7 \%$ of scenarios and there were scenarios where the deterministic findings could be definitive (i.e., an ICER $>£ 30,000 / Q A L Y$ ) but the probabilistic analysis would

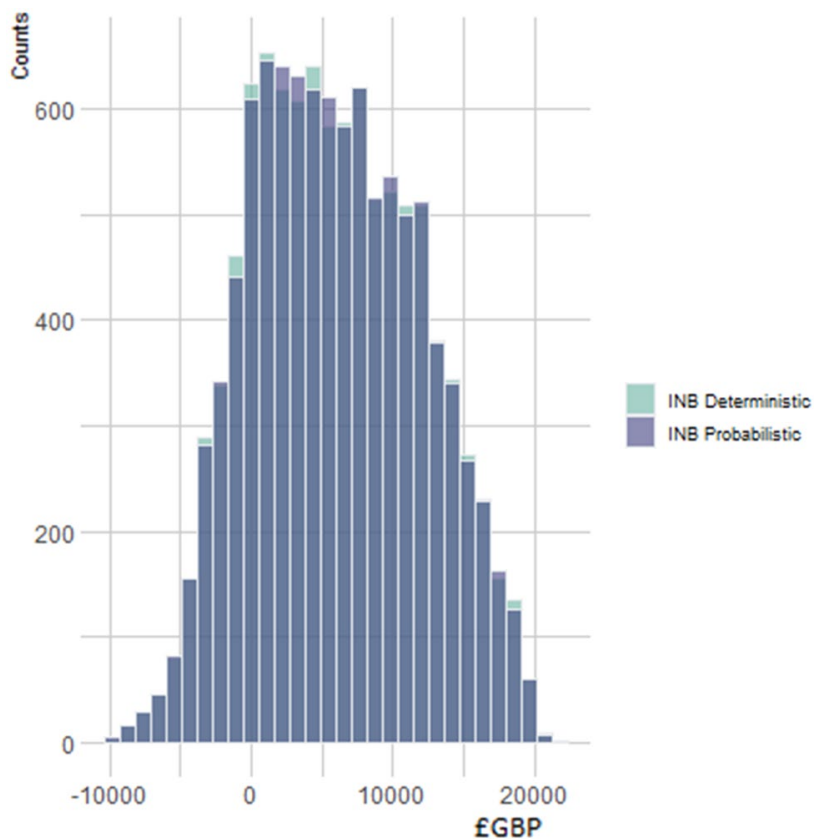

Fig. 1 Histogram of incremental net benefit (INB) estimated by deterministic and probabilistic analysis across 10,000 scenarios*. *Across scenarios the mean and $95 \%$ range of INB deterministic was GBP-2324.80 (- 12688.89, 9367.81), INB probabilistic was GBP6028.64 (- 4363.49, 17688.01), and probabilistic minus deterministic was GBP8353 (7955.72, 8731.12) giving definite evidence of a non-zero bias

indicate substantial uncertainty. The net benefit was found to be a more stable summary of cost-effectiveness than an ICER, so should be preferred in general.

Of course, there are limitations to this analysis. I considered only two treatment options and three health states and only a limited range of distributions for efficacy, cost and utility parameters. There was also no correlation between parameters. However, this analysis represents almost a minimum level of complexity for a Markov model. A greater range of model structures and parameter distributions could be explored in future simulation studies, and it is plausible that deterministic analyses will fail more frequently in more realistic cases.

\section{Recommendation}

The recommendation is to avoid basing decision making on deterministic analysis when using a Markov model. This extends to any non-linear model such as a partitioned survival model or moderately complex decision tree. Even sensitivity analyses should not be deterministic as simply switching from probabilistic to deterministic can affect results and conclusions. 
Supplementary Information The online version contains supplementary material available at https://doi.org/10.1007/s40258-021-00700-1.

\section{Declarations}

Funding This work was funded by the UK Medical Research Council grant MR/S036709/1.

Conflict of interest I have received consulting fees from Novartis Pharma AG, Pfizer Inc, Roche Holding AG, Eisai Inc, Argenx, Janssen Pharmaceutical and BMS. None is directly related to this work.

Data and Code Availability All data and code used in this paper are provided in the supplementary material.

Acknowledgements This work emerged from an issue panel I chaired for the International Society for Pharmcoeconomics and Outcomes Research (ISPOR) European congress in 2020. It was entitled "Uncertainty and Healthcare Decision Making: A Dangerous Combination?" I would like to thank my panellists Jeroen Jansen of University of California in USA, Stephane Regnier of Lundbeck in Switzerland, and Sabine Grimm of Masstricht University Medical Centre in Belgium. I give huge thanks to Dr Sanchez Alvarez at Hoffmann-La Roche who identified an error in the $\mathrm{R}$ code; this was corrected in a correction.

Ethics approval Not applicable.

Consentto participate Not applicable.

Consent for publication Not applicable.

Open Access This article is licensed under a Creative Commons Attribution-NonCommercial 4.0 International License, which permits any non-commercial use, sharing, adaptation, distribution and reproduction in any medium or format, as long as you give appropriate credit to the original author(s) and the source, provide a link to the Creative Commons licence, and indicate if changes were made. The images or other third party material in this article are included in the article's Creative Commons licence, unless indicated otherwise in a credit line to the material. If material is not included in the article's Creative Commons licence and your intended use is not permitted by statutory regulation or exceeds the permitted use, you will need to obtain permission directly from the copyright holder. To view a copy of this licence, visit http://creativecommons.org/licenses/by-nc/4.0/.

\section{References}

1. Briggs AH, Claxton K, Sculpher MJ. Decision modelling for health economic evaluation. Oxford: Oxford University Press; 2006.

2. National Institute for Health and Care Excellence: Guide to the methods of technology appraisal. Process and methods guides, https://www.nice.org.uk/process/pmg9. 2013.

3. CADTH: Procedures for CADTH Reimbursement Reviews. https://cadth.ca/sites/default/files/Drug_Review_Process/ CADTH_Drug_Reimbursement_Review_Procedures.pdf. 2021.

4. Welton NJ, Sutton AJ, Cooper NJ, Abrams KR, Ades AE: Evidence Synthesis for Decision Making in Healthcare: John Wiley and Sons; 2012.

5. Mood AM, Graybill FA, Boes DC: Introduction to the Theory of Statistics: Third Edition: McGraw-Hill International Editions; 1974.

6. Jensen JLWV. Sur les fonctions convexes et les inégalités entre les valeurs moyennes. Acta Math. 1906;30(1):175-93.

7. NICE: TA607: Rivaroxaban for preventing atherothrombotic events in people with coronary or peripheral artery disease. https://www.nice.org.uk/guidance/ta607. 2019.

8. Wilson ECF. Methodological note: reporting deterministic versus probabilistic results of markov, partitioned survival and other non-linear models. Appl Health Econ Health Policy. 2021;19(6):789-95.

9. Incerti D, Thom H, Baio G, Jansen JP. R you still using excel? the advantages of modern software tools for health technology assessment. Value Health. 2019;22(5):575-9.

10. R Core Team: R: A Language and Environment for Statistical Computing. https://www.R-project.org/. Vienna, Austria: R Foundation for Statistical Computing; 2019. 